\title{
ADAPTIVE OPTICS PHOTOMETRY AND ASTROMETRY OF BINARY STARS. III. A FAINT COMPANION SEARCH OF O-STAR SYSTEMS*
}

\author{
Nils H. Turner ${ }^{1}$, Theo A. ten Brummelaar ${ }^{1}$, Lewis C. Roberts ${ }^{2}$, Brian D. Mason ${ }^{3}$, William I. Hartkopf ${ }^{3}$, and \\ Douglas R. GiEs ${ }^{4}$ \\ ${ }^{1}$ Center for High Angular Resolution Astronomy, Georgia State University, The CHARA Array, Mount Wilson, CA 91023, USA; nils@ chara-array.org, \\ theo@ chara-array.org \\ 2 The Boeing Company, 535 Lipoa Pkwy, Ste 200 Kihei HI 96753, USA ${ }^{5}$; lewis.c.roberts@jpl.nasa.gov \\ ${ }^{3}$ United States Naval Observatory, Department of Astrometry, Washington DC, USA; bdm@usno.navy.mil, wih@usno.navy.mil \\ ${ }^{4}$ Center for High Angular Resolution Astronomy, Department of Physics and Astronomy, Georgia State University, P.O. Box 4106, Atlanta, GA 30302-4106, USA; \\ gies@chara.gsu.edu \\ Received 2008 March 18; accepted 2008 April 21; published 2008 June 24
}

\begin{abstract}
We present the results of an adaptive optics survey for faint companions among Galactic O-type star systems (with $V \lesssim 8$ ) using the Advanced Electro-Optical System (AEOS) $3.6 \mathrm{~m}$ telescope on Haleakala. We surveyed these $\mathrm{O}$-star systems in the $I$-band, typically being able to detect a companion with a magnitude difference of $\Delta m_{I} \lesssim 6$ in the projected separation range $0^{\prime \prime} .5<\rho<1^{\prime \prime} 0$, and $\Delta m_{I} \lesssim 9.5$ in the range $1^{\prime \prime} .0<\rho<5$.'0. In the course of the survey, we discovered 40 new companions among 31 of the 116 objects examined and made astrometric and differential magnitude measurements of 24 additional known pairs, several of them being confirmation detections. We present new astrometric orbits for two binaries, BU 1032AB (WDS 05387-0236; $\sigma$ Ori AB) and SEE 322 (WDS 17158-3344; HD 155889AB). We lack magnitude differences for other filter bands, so it is difficult to determine physical from line-of-sight companions, but we present empirical arguments for the limiting magnitude difference where field contamination is significant.
\end{abstract}

Key words: binaries: close - binaries: general - instrumentation: adaptive optics - stars: early-type - techniques: high angular resolution

Online-only material: extended figure set

\section{INTRODUCTION}

Binary star systems are frequently the only source for the fundamental determination of the most basic property of a star, its mass. The masses of a sample of stars, when determined with sufficient accuracy, serve as crucial tests of our theoretical understanding of stellar formation, structure, and evolution. By combining information from the angular orbit projected on the sky (or from a light curve in the case of an eclipsing system) with the spectroscopic orbit, one can determine the distance and masses of the individual stars. In the case of the most massive stars, accurate masses are even more important, not only because massive stars are relatively rare, but because these stars (initial masses $>20 M_{\odot}$ ) play critical roles in galaxies. Massive stars serve as signposts of star formation in galaxies (Massey et al. 1995). Current theory suggests that high-mass stars are born in loosely bound star clusters which disperse after a few Myr. These birth conditions suggest that high-mass stars might often have companions as, for example, found around $\gamma^{2}$ Velorum (Pozzo et al. 2000). Some of these could be very close and gravitationally bound to the $\mathrm{O}$ star, which is suggested by the prevalence of binary and triple systems among young $\mathrm{O}$ stars (Mason et al. 1998).

Mason et al. (1998) performed a speckle interferometry survey of Galactic O-type stars for close companions, specifically looking for differences in the multiplicity frequencies amongst

\footnotetext{
* Based on observations made at the Maui Space Surveillance System operated by Detachment 15 of the US Air Force Research Laboratory's Directed Energy Directorate.

5 Current address, Jet Propulsion Laboratory, 4800 Oak Grove Drive, Pasadena CA 91109, USA.
}

the cluster, field, and runaway O-type star populations, as well as the distribution of orbital periods. They did their survey in the $V$-band using speckle interferometry, which is sensitive to the detection of a companion if the projected separation is in the range $0^{\prime \prime} 035<\rho<1^{\prime \prime} .5$ and the magnitude difference $\Delta m_{V}<3$. Our survey is in the $I$-band (which will slightly emphasize redder companions) and extends the dynamic range of their work. Due to the availability of only one photometric band for this survey, we are unable to determine physical associations for the new companions with any certainty, and we can only address the likelihood that perhaps some of the companions are gravitationally bound. The results presented here invite further investigation into the $\mathrm{O}$ stars and their potential to shed light on star-formation processes.

\section{OBSERVATIONS}

The data were taken using the adaptive optics (AO) system and Visible Imager (VisIm) camera of the Advanced ElectroOptical System (AEOS) $3.6 \mathrm{~m}$ telescope at the Maui Space Surveillance System, located on Haleakala. The data were collected during four separate observing runs between 2001 February and 2002 September. In addition to the dedicated observing runs, some observations were taken as part of a queuescheduled observation program between 2001 May and 2005 November.

\subsection{AEOS Telescope and Adaptive Optics System}

The AEOS telescope is an altitude-azimuth (alt-az) design. While the telescope is very flexible and supports several different optical configurations (Roberts \& Neyman 2002), of interest here is the Cassegrain configuration which feeds a $726 \mathrm{~m}$ focal 
length, $f / 200$ beam (with a field of view (FOV) of 61".9) through a Nasmyth port coudé path to a fold flat below the telescope which directs the beam onto a custom optical table that supports the main components of the $\mathrm{AO}$ system. For this project, the short-wavelength light $(400-700 \mathrm{~nm})$ is sent to the wavefront sensor (WFS) and tracker camera beam trains, while the $700 \mathrm{~nm}-5 \mu \mathrm{m}$ light is sent to the VisIm, which is used as the science detector.

The heart of the AO system is a Shack-Hartmann WFS driving a 941-actuator Xinetics deformable mirror (DM) with a stroke of $\pm 4 \mu \mathrm{m}$. Optics inside the WFS produce a pupil image on a Hartmann lens array. Each generated Hartmann spot is then imaged onto a $4 \times 4$ group of pixels on the WFS CCD. These pixel values can be used directly or binned (for higher throughput) to generate the wavefront slopes. The WFS CCD is a Lincoln Labs frame-transfer device with 16 output ports, capable of frame rates of $0.2-2.5 \mathrm{kHz}$. The wavefront slopes are calculated from the Hartmann spot values by means of many digital signal processors working in parallel, feeding these data to a system which reconstructs the wavefront, taking into account alt-az-induced image rotation and WFS-to-DMactuator mapping artifacts.

The VisIm camera is described in detail in Roberts \& Neyman (2002). In short, it operates from 700 to $1050 \mathrm{~nm}$; is equipped with an atmospheric dispersion corrector; has a two-mode image derotator (zenith at a fixed position in the image or celestial north at a fixed position in the image); and, for this project, has a $10^{\prime \prime}$ FOV $\left(0^{\prime} 020\right.$ pixel $\left.^{-1}\right)$. The detector is a $512 \times 512$ pixel EEV $\mathrm{CCD}$ with a dark current of $22 e^{-}$pixel $^{-1} \mathrm{~s}^{-1}$ (at $-40^{\circ} \mathrm{C}$ ) and a read noise of $12 e^{-} \mathrm{rms}$. The camera output is digitized to 12 bits with $10 e^{-}$per digital number.

\subsection{Object Selection}

The object list started out as all the components in the 228 systems listed in Mason et al. (1998). They were then culled for the effective magnitude limit of the AEOS AO system (about $m_{V}=8$ ), and for the declination limit of acceptable AO correction (objects that at some time during the year get above $30^{\circ}$ elevation at Haleakala, i.e., a declination greater than about $-45^{\circ}$ ). This reduced the list to 164 objects. We added seven more targets from the Galactic O Star Catalog ${ }^{6}$ (MaízApellániz et al. 2004) that are within our adopted magnitude and declination limits. We actually observed, at least once, 116 of the 171 objects in our list. These observations are fairly consistently distributed among the three O-star populations, sampling 63\% of the cluster membership stars, $78 \%$ of the field stars, and $88 \%$ of the runaway stars in our list of potential targets.

\subsection{Data Collection and Reduction}

Since VisIm is only a 12-bit camera, a stellar image can overflow the digitizer in a rather short exposure time. If the frame saturates, then it is omitted from the final image. Frequently, this saturated frame will represent a period of particularly good seeing. In order to keep as many of these "good seeing" frames as possible, we set the exposure time such that the average peak value was about $75 \%$ of the full-well depth. We then built up the signal-to-noise ratio by taking many frames, then weighting and summing them. Figure 1 shows the effect of this summing on the detectable magnitude difference for 1, 10, 50, 100, 250, and 1000 frame(s). On the basis of this plot, we aimed to take at least

\footnotetext{
6 http://www-int.stsci.edu/ jmaiz/research/GOS/GOSmain.html.
}



Figure 1. Assuming that a Gaussian-shaped peak, $3 \sigma$ detection above background constitutes a positive detection of a companion, this plot shows the rough dynamic range of the AEOS AO system as a function of radius for different numbers of summed images. From top to bottom, the curves represent the results of 1000, 250, 100, 50,10, and 1 summed frame(s). The "shelf," most apparent in the upper curves from about $0.7-1$ arcsec in radius, is due to AO system artifacts.

1000 frames of each object, although time constraints, weather, and object brightness occasionally limited us to a lower-frame count.

The VisIm is a frame-transfer camera and is therefore unable to take a bias frame. To compensate, a bias frame was created by taking many dark exposures at a variety of exposure times ranging from $10 \mathrm{~ms}$ to several minutes. A linear fit was made to the signal from each pixel as a function of exposure time; the $y$-intercepts became the bias frame while the slopes became the dark frame. Prior to 2005, flat-field frames were created using the twilight sky to evenly illuminate the VisIm detector. Roberts (2001) found that sky flat-field frames varied at about the $1 \%$ level, so after 2005 , flat-field frames were generated using an internal calibration sphere; this lowered the frame-toframe variation below the $0.01 \%$ level. From this point, all data frames were debiased, dark subtracted, and flat fielded in the conventional way.

For a given sequence of data frames, a weighted shift-andadd algorithm was used to create the final image. The weighted shift-and-add algorithm (ten Brummelaar et al. 1998) is a modification of the traditional image-stacking algorithm that takes seeing conditions in each individual frame into account. Frames with higher peak values (which represent better seeing) influence the final image (by means of weighting) more than frames with lower peak values.

The fitting algorithm is described in detail in ten Brummelaar et al. (1996, 2000). In short, the point-spread function (PSF) used to represent the system performance is that of the primary star in the image, with a few modifications; near the primary, the PSF is a pixel-for-pixel table of numbers, while farther out the PSF is a radially-symmetric series of values. This PSF is fitted to the primary and secondary components and iterated until the intensity ratio converges.

\subsection{Detection Sensitivity}

Figure 1 only gives a qualitative sense of the companion detection sensitivity of the system. Cross comparisons of the reduced images, especially of the same object taken on different nights, show different detection limits. This is primarily due to variations in $\mathrm{AO}$ performance which is primarily attributed 


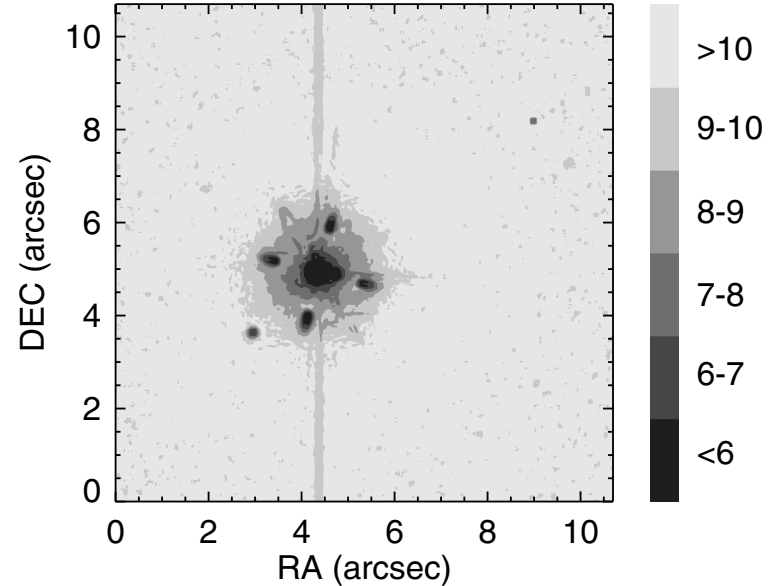

Figure 2. A sample dynamic range map (in this case, HD 34656, taken Bess. year 2003.7360). The gradient map on the right shows the $5 \sigma$ detection limit for a given pixel of the image in magnitudes. There are several artifacts in this image which lower the dynamic range. The largest is the vertical line, which is an artifact of the shutter-less frame transfer process. There are also four waffle spots caused by the Fried geometry of the WFS. There are also hints of the diffraction pattern caused by the secondary mirror support spiders. These all increase the difficulty of finding any companions that coincide with these artifacts.

(An extended figure set is available in the online journal.)

to variations in the atmospheric turbulence profile. In order to quantify the sensitivity of the reduced images, we have created a variation of the "dynamic range map" technique described in Hinkley et al. (2007) which defines the dynamic range of a given position in a $2 \mathrm{D}$ image as the faintest companion detectable at that position to the $5 \sigma$ level. In our version, we construct a map the same size as each reduced image. The intensity level of each pixel in the map is set to five times the root mean square (rms) intensity variation across a patch centered on the corresponding pixel in the original image. The patch is a square with lengths equal to the FWHM of the original image. This produces the dynamic range map in intensity terms, which are then converted to magnitudes.

Figure Set 2 shows the results of this technique applied to all of the images generated in this survey. It is apparent from the sample figure that the detection threshold for a companion is highly spatially variant. The dynamic range increases with increasing radius from the central star. There are several artifacts in this image which lower the dynamic range. The largest is the vertical line, which is an artifact of the shutter-less frametransfer process. There are also four waffle spots caused by the Fried geometry of the WFS (Makidon et al. 2005). There are also hints of the diffraction pattern caused by the secondary mirror support spiders. As the AO system performance decreases, the FWHM of the PSF will increase, which increases the area over which the central PSF causes confusion. In addition, it also widens the companion's PSF, lowering the contrast between the two and decreasing the detection rate. After 2003, the waffle spots were greatly reduced when new reconstructors were put in place to filter out the unsensed waffle modes.

These maps have several purposes. For those objects with multiple observations where companions are seen in some but not all of the data, changes in the dynamic range can help explain the reasons for these discrepancies. They also illustrate the limitations of the data, showing where it is almost impossible to find faint companions. This is often useful when trying to detect known long-period spectroscopic companions.

\section{RESULTS}

\subsection{New Pairs and New Measures}

Table 1 lists the 40 new and 24 known pairs detected in the survey. Four of the new companions (HD 17505, HD 37468, HD 193793, and HD 217086) are in systems with previously known components in the AEOS FOV. The remainder are new companions in systems not previously known to be multiple (at least in the AEOS FOV). The table has nine columns which summarize the information gleaned from the measurements: Column 1 lists the Washington Double Star (WDS) identifier, Columns 2 and 3 identify the object by HD and Hipparcos number, and Column 4 gives the discoverer designation (TRN for new pairs). Circumstances of the observation are given in Columns 5-8. Column 5 gives the Besselian epoch of the start of the observation, Columns 6 and 7 give the position angle (P.A.) (in degrees, where north is $0^{\circ}$ and east is $90^{\circ}$ ) and separation (in seconds of arc) between the primary (the survey star-the brightest star in the image) and the detected component. Note that for many of the objects, the P.A. is missing or ambiguous. The VisIm derotator is usually in one of the two modes, one which keeps zenith up in the image, and one which keeps north up in the image. In the observations with more than one or a missing P.A., the state of the derotator was unknown, leaving the orientation of the image on the sky unknown. However, in some cases, the position of a known pair resolves the ambiguity while for the rest, new observations will be required. Column 8 gives the magnitude difference at the $I$-band between the primary and the detected component. Finally, Column 9 lists the spectroscopic status. Given the separations detected here, none of the short period spectroscopic pairs is expected to be detected. However, listing the spectroscopic binaries helps to form the complete multiplicity picture. The indicator in this table is $\mathrm{V}$ (velocity variable) if one or more close spectroscopic companions is indicated or $\mathrm{C}$ (velocity constant) if radial velocity work indicates no close companion. The spectroscopic reference is generally given in Mason et al. (1998).

The differential magnitude errors in Column 8 of Table 1 were generated from simulated binary data that we analyzed using the same fitting algorithm applied to the measured binary data. To generate the simulated binaries, we started by collecting all the single star images from this survey (see Table 4) and those of a duplicity survey B stars carried out contemporaneously (see Roberts et al. 2007, Table 2). We omitted single-star images where there were fewer than 250 frames used to make the final image. This exercise gave us 167 unique images with PSFs ranging from 0 '.083 to 0.445 , with a decided emphasis on values less than $0^{\prime \prime} 2$. For each entry in Table 1, 167 simulated binaries were constructed using the magnitude difference in Column 8 and the pixel positions of the primary and secondary components in the main image. We then ran the fitting algorithm on each of these 167 simulated images for each table entry. We calculated the magnitude variance in two passes, the first to filter out outliers on the basis of the variance of the fitted pixel separation of the simulated binary (namely, any deviation greater than $1 \sigma$ ), and the second to use this reduced list of simulated fits to generate the final magnitude variance. In general, the number of outliers per table entry was less than 10 . The errors quoted in Column 8 are the standard errors of these variances. Errors are not quoted for the separation (Column 7), but in all cases, the standard error is less than $0{ }^{\prime} 04$, usually less than 0. '005. For errors in P.A. (Column 6), we adopt the values from Roberts et al. (2007), $\pm 2^{\circ}$ for separations less than $1^{\prime \prime}$, and $\pm 1^{\circ}$ for 
Table 1

Survey Measurements

\begin{tabular}{|c|c|c|c|c|c|c|c|c|c|c|}
\hline $\begin{array}{l}\text { WDS } \\
\text { (1) }\end{array}$ & $\begin{array}{l}\text { HD } \\
(2)\end{array}$ & $\begin{array}{l}\text { HIP } \\
(3)\end{array}$ & & $\begin{array}{r}\text { Discov } \\
\text { designa } \\
(4) \\
\end{array}$ & & $\begin{array}{c}\text { Epoch } \\
\text { (BY) } \\
(5)\end{array}$ & $\begin{array}{l}\text { P.A. } \\
\left({ }^{\circ}\right) \\
(6) \\
\end{array}$ & $\begin{array}{c}\text { Separation } \\
(\text { (") } \\
(7) \\
\end{array}$ & $\begin{array}{c}\Delta m_{I} \\
(\mathrm{mag}) \\
(8)\end{array}$ & $\begin{array}{c}\text { Spectroscopic } \\
\text { status }^{\mathrm{c}} \\
(9)\end{array}$ \\
\hline $00061+6341$ & 108 & 505 & TRN & 7 & & 2001.7450 & $5 \mathrm{Z} / 176 \mathrm{~N}^{\mathrm{a}}$ & 3.24 & $9.47 \pm 0.26$ & $\mathrm{C}$ \\
\hline $00177+5126$ & 1337 & 1415 & TRN & 8 & & 2001.7341 & $22 \mathrm{Z} / 186 \mathrm{~N}^{\mathrm{a}}$ & 2.75 & $9.30 \pm 0.47$ & $\mathrm{~V}$ \\
\hline $02158+5600$ & 13745 & 10541 & TRN & 9 & & 2004.7924 & 29 & 4.55 & $10.04 \pm 0.67$ & $\mathrm{~V}$ \\
\hline $02229+4129$ & 14633 & 11099 & TRN & 10 & & 2001.7451 & $97 \mathrm{Z} / 214 \mathrm{~N}^{\mathrm{a}}$ & 2.18 & $10.31 \pm 0.61$ & $\mathrm{~V}$ \\
\hline \multirow{5}{*}{$02327+6127$} & 15558 & 11832 & TRN & 11 & $\mathrm{AF}$ & 2001.7450 & 261 & 4.45 & $6.59 \pm 0.19$ & V \\
\hline & & & & & & 2002.6845 & 261 & 4.48 & $6.38 \pm 0.40$ & \\
\hline & & & & & & 2005.6962 & 262 & 4.44 & $6.42 \pm 0.30$ & \\
\hline & & & TRN & 11 & AG & 2001.7450 & 221 & 2.99 & $9.94 \pm 0.35$ & \\
\hline & & & TRN & 11 & $\mathrm{AH}$ & 2001.7450 & 211 & 5.79 & $11.03 \pm 0.86$ & \\
\hline $02407+6117$ & 16429 & 12495 & TRN & 12 & $\mathrm{AD}$ & 2005.6963 & 112 & 2.94 & $7.39 \pm 0.22$ & $\mathrm{~V}$ \\
\hline \multirow[t]{6}{*}{$02511+6025$} & 17505 & 13296 & STF & 306 & $\mathrm{AB}$ & 2001.7450 & 91 & 2.09 & $1.71 \pm 0.26$ & V \\
\hline & & & & & & 2002.6845 & 89 & 2.11 & $1.71 \pm 0.37$ & \\
\hline & & & & & & 2004.9648 & 92 & 2.10 & $1.64 \pm 0.37$ & \\
\hline & & & TRN & 13 & $\mathrm{AH}$ & 2001.7450 & 142 & 4.59 & $6.72 \pm 0.21$ & \\
\hline & & & & & & 2002.6845 & 140 & 4.62 & $7.01 \pm 0.27$ & \\
\hline & & & & & & 2004.9648 & 143 & 4.59 & $7.15 \pm 0.22$ & \\
\hline $02594+6034$ & 18326 & 13924 & TRN & 14 & & 2001.7451 & $223 \mathrm{Z} / 353 \mathrm{~N}^{\mathrm{a}}$ & 2.38 & $7.45 \pm 0.46$ & $\mathrm{~V}$ \\
\hline $03141+5934$ & 19820 & 15063 & TRN & 15 & & 2001.7452 & $81 \mathrm{Z} / 209 \mathrm{~N}^{\mathrm{a}}$ & 2.81 & $10.37 \pm 0.42$ & V \\
\hline $03556+5238$ & 24431 & 18370 & HDS & 494 & & 2001.7455 & 176 & 0.71 & $2.86 \pm 0.35$ & $\mathrm{C}$ \\
\hline $03590+3548$ & 24912 & 18614 & TRN & 16 & & 2001.7342 & $141 \mathrm{Z} / 236 \mathrm{~N}^{\mathrm{a}}$ & 2.40 & $9.81 \pm 1.16$ & $\mathrm{C}$ \\
\hline $05163+3419$ & 34078 & 24575 & TRN & 17 & $\mathrm{Aa}$ & 2004.7873 & 171 & 0.35 & $4.97 \pm 0.51$ & $\mathrm{C}$ \\
\hline \multirow[t]{8}{*}{$05207+3726$} & 34656 & 24957 & TRN & 18 & $\mathrm{Aa}$ & 2002.7257 & 279 & 0.35 & $3.77 \pm 0.33$ & $\mathrm{C}$ \\
\hline & & & & & & 2003.7360 & 280 & 0.35 & $4.09 \pm 1.10$ & \\
\hline & & & & & & 2003.8917 & 280 & 0.34 & $4.13 \pm 0.84$ & \\
\hline & & & TRN & 18 & $\mathrm{AB}$ & 2002.7257 & 48 & 1.88 & $6.98 \pm 0.24$ & \\
\hline & & & & & & 2003.7360 & 47 & 1.90 & $6.94 \pm 0.25$ & \\
\hline & & & & & & 2003.8917 & 47 & 1.89 & $7.28 \pm 0.43$ & \\
\hline & & & TRN & 18 & $\mathrm{AC}$ & 2003.7360 & 126 & 2.96 & $10.57 \pm 0.68$ & \\
\hline & & & TRN & 18 & $\mathrm{AD}$ & 2003.7360 & 247 & 5.75 & $10.39 \pm 0.42$ & \\
\hline $05297+3523$ & 35921 & 25733 & $\mathrm{HU}$ & 217 & & 2004.7873 & 253 & 0.61 & $2.23 \pm 0.31$ & $\mathrm{~V}$ \\
\hline \multirow{2}{*}{$05351+0956$} & 36861 & & STF & 738 & $\mathrm{AB}$ & 2001.7428 & $297 \mathrm{Z} / 356 \mathrm{~N}^{\mathrm{a}, \mathrm{b}}$ & 4.25 & $2.29 \pm 0.16$ & $\mathrm{C}$ \\
\hline & & & & & & 2001.7456 & 41 & 4.23 & $2.04 \pm 0.15$ & \\
\hline \multirow[t]{2}{*}{$05353-0523$} & 37022 & 26221 & STF & 748 & $\mathrm{Ca}, \mathrm{F}$ & 2001.7456 & 118 & 4.39 & $4.89 \pm 1.06$ & $\mathrm{~V}$ \\
\hline & & & & & & 2003.0045 & 120 & 4.46 & $4.73 \pm 0.19$ & \\
\hline $05354-0525$ & 37041 & 26235 & CHR & 249 & Aa & 2004.8666 & 292 & 0.39 & $2.97 \pm 0.72$ & $\mathrm{~V}$ \\
\hline \multirow[t]{2}{*}{$05387-0236$} & 37468 & 26549 & $\mathrm{BU}$ & 1032 & $\mathrm{AB}$ & 2001.7456 & 106 & 0.24 & $1.22 \pm 2.15$ & V \\
\hline & & & TRN & 19 & $\mathrm{AF}$ & 2001.7456 & 18 & 3.13 & $8.07 \pm 0.33$ & \\
\hline $05407-0157$ & 37742 & & STF & 774 & Aa-B & 2001.7455 & 160 & 2.36 & $2.24 \pm 0.89$ & $\mathrm{C}$ \\
\hline $06319+0457$ & 46150 & 31130 & GAN & 3 & $\mathrm{AB}$ & 2001.0989 & 284 & 3.47 & $4.87 \pm 0.13$ & $\mathrm{C}$ \\
\hline $06322+0450$ & 46223 & 31149 & TRN & 20 & & 2001.8661 & $314 \mathrm{Z} / 264 \mathrm{~N}^{\mathrm{a}}$ & 0.46 & $4.37 \pm 0.17$ & $\mathrm{C}$ \\
\hline $06364+0605$ & 46966 & 31567 & TRN & 21 & & 2002.8023 & 210 & 3.19 & $9.97 \pm 0.63$ & $\mathrm{C}$ \\
\hline \multirow{3}{*}{$06374+0608$} & 47129 & 31646 & TRN & 22 & $\mathrm{AB}$ & 2002.2404 & 240 & 1.15 & $5.10 \pm 0.43$ & $\mathrm{~V}$ \\
\hline & & & & & & 2005.0144 & 251 & 1.16 & $4.94 \pm 0.22$ & \\
\hline & & & TRN & 22 & $\mathrm{AC}$ & 2005.0144 & 203 & 0.78 & $5.14 \pm 0.22$ & \\
\hline $06386+0137$ & 47432 & 31766 & TRN & 23 & & 2005.0134 & 206 & 0.78 & $4.95 \pm 1.31$ & $\mathrm{C}$ \\
\hline $06410+0954$ & 47839 & 31978 & STF & 950 & Aa-B & 2001.7457 & 202 & 2.88 & $3.31 \pm 2.11$ & $\mathrm{~V}$ \\
\hline $06427+0143$ & 48279 & 32137 & STF & 956 & $\mathrm{AB}$ & 2004.0478 & 199 & 6.63 & $2.57 \pm 0.39$ & $\mathrm{C}$ \\
\hline $08392-4025$ & 73882 & 42433 & $\mathrm{~B}$ & 1623 & & 2004.1190 & 256 & 0.65 & $1.25 \pm 0.77$ & $\mathrm{~V}$ \\
\hline $16550-4109$ & 152408 & 82775 & I & 576 & & 2001.4030 & $260 \mathrm{Z} / 261 \mathrm{~N}^{\mathrm{a}}$ & 5.30 & $5.67 \pm 0.92$ & $\mathrm{C}$ \\
\hline $17065-3527$ & 154368 & 83706 & B & 894 & & 2002.6730 & 357 & 2.57 & $6.28 \pm 0.54$ & $\mathrm{~V}$ \\
\hline $17158-3344$ & 155889 & 84444 & SEE & 322 & & 2003.6041 & 285 & 0.19 & $0.68 \pm 2.59$ & $\mathrm{C}$ \\
\hline \multirow[t]{2}{*}{$17175-2746$} & 156212 & 84588 & TRN & 24 & $\mathrm{AB}$ & 2001.4959 & $206 \mathrm{Z} / 204 \mathrm{~N}^{\mathrm{a}}$ & 4.04 & $8.05 \pm 0.39$ & $\mathrm{C}$ \\
\hline & & & TRN & 24 & $\mathrm{AC}$ & 2001.4959 & $145 \mathrm{Z} / 144 \mathrm{~N}^{\mathrm{a}}$ & 7.31 & $7.40 \pm 0.24$ & \\
\hline $17347-3235$ & 159176 & 86011 & HDS & 2480 & $\mathrm{Ab}$ & 2003.3280 & 59 & 0.70 & $3.14 \pm 0.47$ & $\mathrm{~V}$ \\
\hline $17595-3601$ & 163758 & & TRN & 25 & & 2001.4960 & $107 \mathrm{Z} / 93 \mathrm{~N}^{\mathrm{a}}$ & 1.70 & $8.05 \pm 0.33$ & $\mathrm{C}$ \\
\hline $18024-2302$ & 164492 & 88333 & TRN & 26 & $\mathrm{AH}$ & 2002.4516 & 342 & 1.48 & $5.12 \pm 0.23$ & V \\
\hline $18026-2415$ & 164536 & & RST & 3149 & $\mathrm{AB}$ & 2002.4627 & 62 & 1.65 & $4.49 \pm 12^{\mathrm{d}}$ & $\mathrm{C}$ \\
\hline $18061-2412$ & 165246 & & B & 376 & & 2002.4819 & 98 & 1.90 & $3.68 \pm 0.47$ & V \\
\hline $18152-2023$ & 167263 & & $\mathrm{BU}$ & 286 & $\mathrm{AB}$ & 2005.6545 & 216 & 5.93 & $5.62 \pm 0.98$ & $\mathrm{~V}$ \\
\hline $18181-1215$ & 167971 & 89681 & TRN & 27 & & 2001.7447 & $80 Z / 39 \mathrm{~N}^{\mathrm{a}}$ & 4.70 & $8.12 \pm 0.32$ & $\mathrm{~V}$ \\
\hline $20035+3601$ & 190429 & 98753 & STF & 2624 & Aa-B & 2001.7337 & 172 & 1.92 & $0.84 \pm 0.95$ & $\mathrm{C}$ \\
\hline \multirow[t]{2}{*}{$20181+4044$} & 193322 & 100069 & STF & 2666 & Aa-B & 2001.6683 & 244 & 2.69 & $2.51 \pm 0.12$ & $\mathrm{~V}$ \\
\hline & & & & & & 2001.7364 & 243 & 2.67 & $2.11 \pm 0.26$ & \\
\hline
\end{tabular}


Table 1

(Continued)

\begin{tabular}{|c|c|c|c|c|c|c|c|c|c|c|}
\hline \multirow{3}{*}{$\begin{array}{l}\text { WDS } \\
\frac{(1)}{20191+3916}\end{array}$} & \multirow{2}{*}{$\begin{array}{c}\text { HD } \\
(2)\end{array}$} & $\begin{array}{c}\text { HIP } \\
\text { (3) }\end{array}$ & \multicolumn{3}{|c|}{$\begin{array}{l}\text { Discoverer } \\
\text { designation } \\
\text { (4) }\end{array}$} & \multirow{2}{*}{$\begin{array}{c}\begin{array}{c}\text { Epoch } \\
\text { (BY) } \\
(5) \\
2001.7367\end{array}\end{array}$} & \multirow{2}{*}{$\begin{array}{l}\text { P.A. } \\
\left({ }^{\circ}\right) \\
(6) \\
78\end{array}$} & \multirow{2}{*}{$\begin{array}{c}\text { Separation } \\
\left({ }^{\prime \prime}\right) \\
(7) \\
4.75\end{array}$} & \multirow{2}{*}{$\begin{array}{c}\begin{array}{c}\Delta m_{I} \\
(\mathrm{mag}) \\
(8)\end{array} \\
5.80 \pm 0.17\end{array}$} & \multirow{2}{*}{$\begin{array}{c}\begin{array}{c}\text { Spectroscopic } \\
\text { status }^{\mathrm{c}} \\
(9)\end{array} \\
\mathrm{C}\end{array}$} \\
\hline & & 100173 & TRN & 28 & $\mathrm{AB}$ & & & & & \\
\hline & & & & & & 2002.6730 & 79 & 4.79 & $6.18 \pm 0.25$ & \\
\hline & & & TRN & 28 & $\mathrm{AC}$ & 2001.7367 & 157 & 3.20 & $7.23 \pm 0.28$ & \\
\hline & & & & & & 2002.6730 & 158 & 3.22 & $7.44 \pm 0.16$ & \\
\hline \multirow[t]{8}{*}{$20205+4351$} & 193793 & 100287 & BU & 1207 & $\mathrm{AB}$ & 2001.3400 & 209 & 4.77 & $8.44 \pm 0.31$ & V \\
\hline & & & & & & 2001.7337 & 209 & 4.77 & $6.75 \pm 0.28$ & \\
\hline & & & & & & 2001.7363 & 209 & 4.77 & $6.75 \pm 12^{\mathrm{d}}$ & \\
\hline & & & & & & 2001.7447 & $\ldots b$ & 4.79 & $6.43 \pm 0.53$ & \\
\hline & & & & & & 2002.6731 & 210 & 4.81 & $6.58 \pm 0.13$ & \\
\hline & & & & & & 2002.6731 & 210 & 4.81 & $6.93 \pm 0.12$ & \\
\hline & & & TRN & 29 & $\mathrm{AC}$ & 2001.7363 & 202 & 2.29 & $9.62 \pm 0.35$ & \\
\hline & & & & & & 2001.7447 & $\ldots^{\mathrm{b}}$ & 3.23 & $9.32 \pm 0.38$ & \\
\hline $20566+4455$ & 199579 & 103371 & TRN & 30 & & 2001.7338 & $30 \mathrm{Z} / 205 \mathrm{~N}^{\mathrm{a}}$ & 3.76 & $9.79 \pm 0.29$ & V \\
\hline $21079+3324$ & 201345 & 104316 & TRN & 31 & & 2001.7367 & $342 \mathrm{Z} / 218 \mathrm{~N}^{\mathrm{a}}$ & 7.38 & $9.31 \pm 0.47$ & $\mathrm{C}$ \\
\hline $21185+4357$ & 203064 & 105186 & TRN & 32 & & 2001.7337 & $81 Z / 219 \mathrm{~N}^{\mathrm{a}}$ & 3.84 & $9.48 \pm 0.29$ & $\mathrm{C}$ \\
\hline $21390+5729$ & 206267 & 106886 & $\mathrm{BU}$ & 1143 & $\mathrm{AB}$ & 2001.7338 & 317 & 1.78 & $5.65 \pm 0.21$ & V \\
\hline $21449+6228$ & 207198 & 107374 & TRN & 33 & $\mathrm{AC}$ & 2001.7364 & $75 \mathrm{Z} / 207 \mathrm{~N}^{\mathrm{a}}$ & 2.96 & $10.43 \pm 0.52$ & $\mathrm{C}$ \\
\hline $22021+5800$ & 209481 & 108772 & TRN & 34 & & 2001.7341 & $358 \mathrm{Z} / 208 \mathrm{~N}^{\mathrm{a}}$ & 2.75 & $9.92 \pm 0.46$ & V \\
\hline \multirow[t]{2}{*}{$22051+6217$} & 209975 & 109017 & TRN & 35 & $\mathrm{AD}$ & 2001.7367 & $341 \mathrm{Z} / 171 \mathrm{~N}^{\mathrm{a}}$ & 4.14 & $9.98 \pm 0.44$ & $\mathrm{C}$ \\
\hline & & & TRN & 35 & $\mathrm{AE}$ & 2001.7367 & $344 \mathrm{Z} / 175 \mathrm{~N}^{\mathrm{a}}$ & 3.79 & $10.03 \pm 0.37$ & \\
\hline $22393+3903$ & 214680 & 111841 & TRN & 36 & $\mathrm{AC}$ & 2001.7339 & $85 \mathrm{Z} / 222 \mathrm{~N}^{\mathrm{a}}$ & 3.56 & $9.94 \pm 0.45$ & $\mathrm{C}$ \\
\hline \multirow[t]{6}{*}{$22568+6244$} & 217086 & 113306 & MLR & 266 & $\mathrm{AB}$ & 2001.7369 & 353 & 2.79 & $3.41 \pm 0.27$ & $\mathrm{C}$ \\
\hline & & & & & & 2002.6843 & 354 & 2.83 & $3.34 \pm 0.48$ & \\
\hline & & & & & & 2004.7598 & 354 & 2.78 & $3.79 \pm 0.58$ & \\
\hline & & & TRN & 37 & $\mathrm{AC}$ & 2001.7369 & 164 & 3.15 & $7.14 \pm 0.29$ & \\
\hline & & & & & & 2002.6843 & 165 & 3.17 & $6.79 \pm 12^{\mathrm{d}}$ & \\
\hline & & & & & & 2004.7598 & 165 & 3.12 & $7.03 \pm 0.34$ & \\
\hline
\end{tabular}

Notes.

${ }^{a}$ Image derotator in an unknown state: $\mathrm{Z}=$ zenith up and $\mathrm{N}=$ north up. Confirmation of the pair will clear up this residual ambiguity.

${ }^{\mathrm{b}}$ Image derotator in an unknown state. Neither zenith up or north up match coordinates of known object in field.

${ }^{\mathrm{c}}$ Codes are $\mathrm{V}$, one or more additional spectroscopic companions indicated or $\mathrm{C}$, radial velocity constant.

${ }^{\mathrm{d}}$ This is a lower limit for the errors, the actual errors are typically much larger.

Table 2

Orbital Elements

\begin{tabular}{|c|c|c|c|c|c|c|c|c|c|c|}
\hline $\begin{array}{l}\text { WDS designation } \\
\quad \alpha \delta(2000) \\
\end{array}$ & & \multicolumn{2}{|c|}{$\begin{array}{l}\text { Discoverer } \\
\text { designation }\end{array}$} & $\begin{array}{l}\text { Period } \\
P(\mathrm{yr}) \\
\end{array}$ & $\begin{array}{c}\text { Semimajor } \\
\text { axis } \\
a\left({ }^{\prime \prime}\right) \\
\end{array}$ & $\begin{array}{l}\text { Inclination } \\
\qquad i(\circ)\end{array}$ & $\begin{array}{c}\text { Longitude } \\
\text { of node } \\
\Omega(0) \\
1217\end{array}$ & $\begin{array}{c}\text { Epoch of } \\
\text { periastron } \\
T_{o}(\mathrm{yr}) \\
\end{array}$ & \multirow{2}{*}{$\begin{array}{c}\text { Eccentricity } \\
\qquad \begin{array}{c}e \\
0.0515 \\
\pm 0.0080\end{array}\end{array}$} & \multirow{2}{*}{ 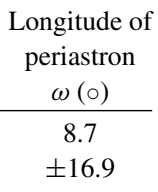 } \\
\hline $05387-0236$ & $\mathrm{BU}$ & 1032 & $\mathrm{AB}$ & $\begin{array}{l}156.7 \\
\pm 3.0\end{array}$ & $\begin{array}{c}0.2662 \\
\pm 0.0021\end{array}$ & $\begin{array}{l}159.7 \\
\pm 3.7\end{array}$ & $\begin{array}{l}121.7 \\
\pm 9.6\end{array}$ & $\begin{array}{l}1999.5 \\
\pm 10.2\end{array}$ & & \\
\hline $05387-0236$ & $\mathrm{BU}$ & 1032 & $\mathrm{AB}^{\mathrm{a}}$ & $\begin{array}{l}155.3 \\
\pm 7.5\end{array}$ & $\begin{array}{c}0.2642 \\
\pm 0.0052\end{array}$ & $\begin{array}{l}160.4 \\
\pm 7.2\end{array}$ & $\begin{array}{l}136 . \\
\pm 25\end{array}$ & $\begin{array}{l}1997 \\
\pm 24\end{array}$ & $\begin{array}{c}0.051 \\
\pm 0.015\end{array}$ & $\begin{array}{c}18 \\
\pm 37\end{array}$ \\
\hline $17158-3344$ & SEE & 322 & & 536. & 0.35 & 111. & 167. & 1870. & 0.64 & 109. \\
\hline
\end{tabular}

Note. ${ }^{a}$ Elements from (Hartkopf et al. 1996).

separations greater than $1^{\prime \prime}$. Two effects lead to this uncertainty, the clocking calibration of the dove prism in the derotator, and the slight natural image rotation during the frame collection sequence when the derotator is in the fixed zenith position mode.

\subsubsection{Notes to Pairs}

Here we discuss the various binary systems in Table 1 by WDS identifier and discovery designation. In the cases of the stars classified as runaways, it is unlikely that the detected companion is physical (see Section 4).

$02229+4129=T R N 10$. This is a runaway star with a close, low-luminosity companion (Boyajian et al. 2005).

$02407+6117=T R N 12 A D$. The A component is a spectroscopic triple (McSwain 2003).

$02511+6025=T R N 13 A H$. The A component of HD 17505 is a spectroscopic triple (Hillwig et al. 2006). It is uncertain if the new component (AH, $\rho \sim 44^{\prime \prime} 6$ ) and the known pair of Table 1 (STF 306AB) are both gravitationally bound. If STF 
$306 \mathrm{AB}$ is indeed gravitationally bound, Hillwig et al. (2006) estimate it to have a period of 27,000 years.

$03556+5238=H D S 494$. The $\triangle m$ was probably a little too large for Mason et al. (1998) to detect this pair, but it was detected in later speckle observations by Mason et al. (2001a).

$03590+3548=T R N$ 16. Classified as a runaway star in Gies \& Bolton (1986).

$05163+3419=$ TRN 17Aa. Classified as a runaway star in Gies \& Bolton (1986). SEI 136AB ( $\rho \sim 8 . \prime 8, \Delta m \sim 3.3$, just outside the FOV) is a likely optical pair, with the B component not physically associated with this close double.

$05207+3726=T R N 18$. One new close pair and three wide pairs are found here. The close one is designated Aa while the wider pairs are designated $\mathrm{AB}, \mathrm{AC}$, and $\mathrm{AD}$. The known pair SEI $201(\rho \sim 24$ ". $7, \triangle m \sim 5.9)$ is outside the FOV and has been designated $\mathrm{AE}$.

$05297+3523=H U 217$. This known, relatively close, pair has been measured since 1900 . The P.A. has only changed by $4^{\circ}$ over this time span.

05353-0523 $=$ STF 748Ca,F. The primary is $\theta^{1}$ Ori $\mathrm{C}$, the most massive star in the Orion Trapezium. It has a closer companion discovered by speckle methods (Kraus et al. 2007).

05354-0525 = CHR 249Aa. Perryman \& ESA (1997) measured a $\triangle H_{p}$ of 3.23. Fabricius \& Makarov (2000) found $\triangle B_{T}=4.37 \pm 0.02$ and $\triangle V_{T}=3.21 \pm 0.01$.

$05387-0236=T R N$ 19AF. The new component (AF) is at a separation nicely nestled between BU $1032 \mathrm{AB}$ and STF $762 \mathrm{AC}$, at a value which would be consistent with a hierarchical arrangement. The new TRN 19AF component is much brighter in the mid-infrared where it was first discovered (van Loon \& Oliveira 2003) and it is probably a low-mass Kstar surrounded by a circumstellar disk (Caballero 2007). See Section 3.1.3 below for a discussion of the orbit of BU 1032AB.

$05407-0157=S T F 774 A a-B$. This pair has an orbit (Hopmann 1967) with residuals of $\mathrm{O}-\mathrm{C}=-5^{\circ}$ and $-0^{\prime \prime} 03$ in P.A. and separation. However, the orbit is classified as "indeterminate" (grade 5) in the 6th Catalog of Orbits of Visual Binary Stars, ${ }^{6}$ so these residuals are not indicative of measurement quality. While the total number of measures has increased by about $50 \%$ since this orbit was published, the initial elements are of such low quality that even at this point any sort of improvement above "indeterminate" subjective quality is not yet possible. Some of the scatter in the residuals may be due to the presence of a closer companion to $\zeta$ Ori A found by interferometry (Hummel et al. 2000).

$06319+0457=G A N 3 A B$. The measure agrees with earlier published data quite well and indicates that the measure made in 1928 (Silbernagel 1931) is probably erroneous.

06410+0954 = STF 950Aa-B. This pair has only been changed by $\sim 9^{\circ}$ and $0^{\prime} .2$ since it was first resolved by Struve in 1825 (Struve 1837).

$16550-4109=$ I 576. I 576 has not been measured since 1934 (Wallenquist 1934), so identification of this pair with that of Innes is uncertain.

$17065-3527=B 894$. This binary, unconfirmed since its first resolution by van den Bos (1928), has closed slightly.

17158-3344 = SEE 322. This pair has shown noticeable Keplerian motion, completing about $70^{\circ}$ of orbital revolution since discovery (See 1898). See Section 3.1.3 below for a discussion of the orbit.

\footnotetext{
7 http://ad.usno.navy.mil/wds/orb6.html.
}

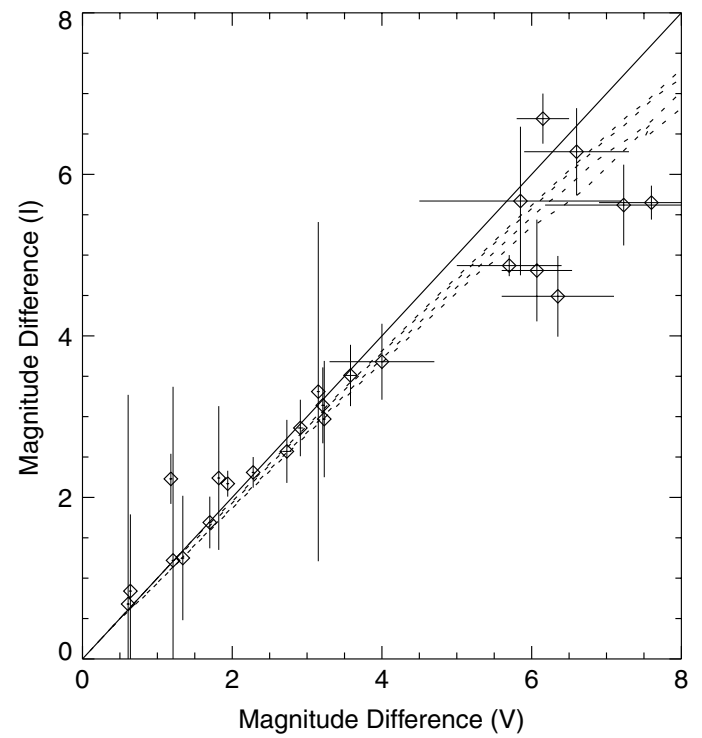

Figure 3. $V$-band magnitude differences from the WDS vs. $I$-band values from $\mathrm{AO}$. The solid line indicates a $1: 1$ relation between $\triangle V$ and $\triangle I$, while the various dotted curves illustrate the expected trend assuming main-sequence primaries of types $\mathrm{O} 3, \mathrm{O} 5, \mathrm{O} 8$, and $\mathrm{B} 0$ (from top to bottom, respectively) and a range of later spectral-type secondaries.

$17347-3235=H D S 2480 A b$. The pairs, ISO 5 and HJ 4962 should have been seen as well. ISO 5 was probably missed due to the corrected FWHM. HJ 4962 was probably just out of the field.

$18024-2302=T R N 26 \mathrm{AH}$. While this pair may be physical, the known double $\mathrm{H} \mathrm{N} 40$ almost certainly is not.

$18026-2415=R S T$ 3149AB. This pair has been seen only twice since 1935. The occultation pair of Africano et al. (1975) was not seen.

$18061-2412=B$ 376. Unconfirmed since its discovery in 1927 (van den Bos 1928). The A component is an eclipsing binary with a $4.6 \mathrm{~d}$ period (Otero 2007).

$20035+3601=S T F 2624 A a-B$. The close pair, MCA 59Aa, which is notoriously difficult to detect, was not seen here.

$20181+4044=S T F 2666 A a-B$. The close speckle pair CHR $96 \mathrm{Aa}$ was not recovered.

$20205+4351=T R N 29 A C$. It is unclear whether there are two new components or a nearby optical pair with a large relative proper motion detected at two different positions.

$21390+5729=B U 1143 A B$. The close Aa pair (MIU 2) was not recovered, although it may have been too close.

$21449+6228=$ TRN 33AC. The visual pair, MLR 16, would have been much too wide $\left(17^{\prime \prime} .6\right)$ to detect here.

$22393+3903=T R N 36 A C$. The visual binary, $\mathrm{S} 813$, at $\sim 1$ arcmin of separation is much too wide to detect here.

$22568+6244=T R N 37 A C$. It is hard to imagine a geometry where both this pair and MLR 266 would be dynamically stable. More than likely one (or both) are optical.

\subsubsection{Comparison of V-and I-Band Magnitude Differences}

Among the O-star primaries listed in the WDS, we may estimate the companion spectral type from the magnitude difference $\Delta V$ for those with small magnitude differences. As the magnitude difference gets larger, the uncertainty in the companion spectral type increases. However, the situation improves with the availability of magnitude differences for two filters. We compare the $V$-band magnitude differences from the WDS with the $I$-band values we determined by AO. The result is shown in Figure 3. Error bars for the visual data were based 


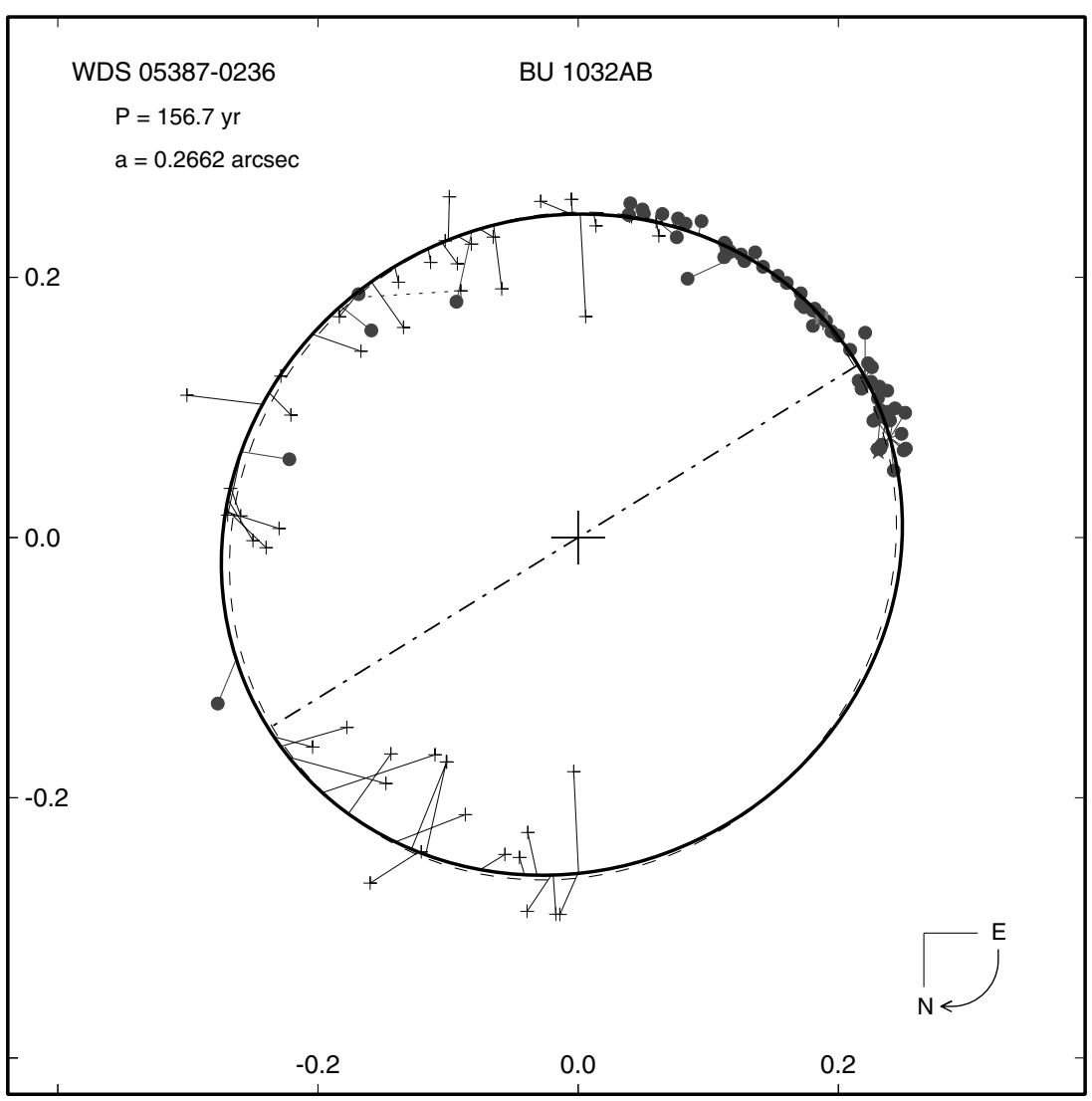

Figure 4. Relative visual orbit of BU 1032; the $x$ and $y$ scales are in arcseconds. The solid curve represents the newly determined orbit (Table 2). The dot-dashed line indicates the line of nodes. High-resolution measurements are shown as filled circles (speckle) or filled stars (AO). Visual measurements are denoted with plus signs. The orientation and direction of motion are indicated in the lower-right corner of the plot. The orbit of Hartkopf et al. (1996) is shown as a dashed curve, which matches the new orbit quite well.

Table 3

Future Ephemerides (BY) for BU 1032 and SEE 322

\begin{tabular}{|c|c|c|c|c|c|c|c|c|c|c|c|c|c|}
\hline \multirow{2}{*}{$\begin{array}{l}\text { WDS designation } \\
\alpha, \delta(2000) \\
05387-0236\end{array}$} & \multirow[b]{2}{*}{ BU } & \multicolumn{2}{|c|}{$\begin{array}{l}\text { Discoverer } \\
\text { designation }\end{array}$} & \multicolumn{2}{|c|}{$\begin{array}{c}2008 \\
\theta\left(^{\circ}\right) \rho\left({ }^{\prime \prime}\right)\end{array}$} & \multicolumn{2}{|c|}{$\begin{array}{c}2010 \\
\theta\left({ }^{\circ}\right) \rho\left({ }^{\prime \prime}\right)\end{array}$} & \multicolumn{2}{|c|}{$\begin{array}{c}2012 \\
\theta\left(^{\circ}\right) \rho\left({ }^{\prime \prime}\right) \\
\end{array}$} & \multicolumn{2}{|c|}{$\begin{array}{c}2014 \\
\theta\left(^{\circ}\right) \rho\left({ }^{\prime \prime}\right) \\
\end{array}$} & \multicolumn{2}{|c|}{$\begin{array}{c}2016 \\
\theta\left(^{\circ}\right) \rho\left({ }^{\prime \prime}\right) \\
\end{array}$} \\
\hline & & 1032 & $\mathrm{AB}$ & 93.0 & 0.249 & 88.0 & 0.249 & 83.1 & 0.248 & 78.1 & 0.247 & 73.1 & 0.246 \\
\hline $17158-3344$ & SEE & 322 & & 281.7 & 0.184 & 280.4 & 0.184 & 279.0 & 0.183 & 277.7 & 0.183 & 276.3 & 0.183 \\
\hline
\end{tabular}

on typical Tycho-2 photometric errors when available (smaller$\triangle m$ pairs), and on scatter among individual visual estimates for those (larger- $\triangle m$ ) systems with more than one such estimate. A rough average value of this scatter $(0.7 \mathrm{mag})$ was used for systems with only one visual $\Delta m_{V}$ estimate. Mean $\Delta m_{I}$ values and error bars are given for systems with multiple observations. For systems with no $I$-band error estimate a default value of 0.5 mag was adopted.

This figure shows that $\Delta V \approx \Delta I$ for small magnitude differences, but $\Delta V$ increases more rapidly at larger values of $\Delta I$. This is as expected for our set of O-star targets: the components of small- $\triangle m$ systems are of similar spectral type, but the secondaries become later in spectral type-hence redder-as $\triangle m$ increases.

To determine the expected extent of this trend, absolute $V$ and I magnitudes for main-sequence spectral types were extracted from Allen's Astrophysical Quantities (Cox 2001). Values of $\triangle V$ and $\triangle I$ were then determined, assuming primaries of spectral types $\mathrm{O} 3, \mathrm{O} 5, \mathrm{O} 8$, and $\mathrm{B} 0$ and secondaries covering a wide range of spectral types. The results, plotted as dotted lines in Figure 3, appear to be in good agreement with the trend seen in these AO observations and are consistent with the assumption that the companions are physical.

\subsubsection{Orbits of $B U 1032$ ( $\sigma$ Ori $A B)$ and SEE 322 (HD 155889)}

Worley (1990) provides guidelines as to when a collection of binary star measures merits the publication of a new orbit. Following these criteria, we determined that two systems, BU 1032 and SEE 322, deserved new orbital solutions. All available measures for these pairs were extracted from the WDS (Mason et al. 2001b) and individual measures were weighted following the precepts of Hartkopf et al. (2001). The orbital elements were determined with an iterative three-dimensional grid-search algorithm (Seymour et al. 2002). For these two pairs, orbital elements are provided in Table 2 and future ephemerides in Table 3. Elements from the Hartkopf et al. (1996) orbit for BU 1032 are provided for comparison. Due to the preliminary nature of the SEE 322 orbit, errors are not quoted. These orbits are illustrated in Figures 4 and 5.

Since the publication of the orbital elements of BU 1032 by Hartkopf et al. (1996), the number of measures has increased by about $35 \%$, and while the prior orbit adequately fits these data, a 


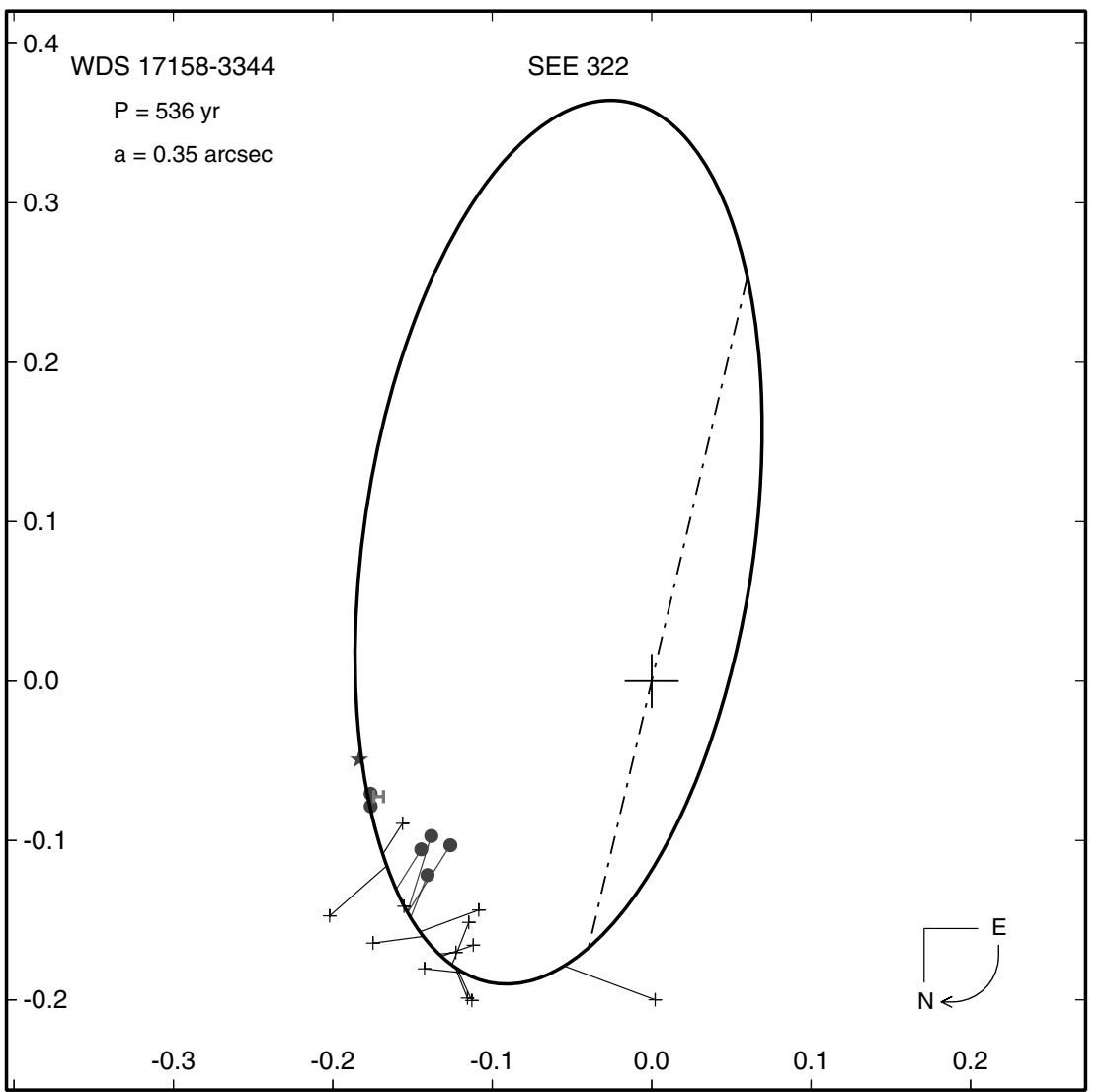

Figure 5. Relative visual orbit for SEE 322 in the same format as Figure 4. The $\mathbf{H}$ indicates the measure of Hipparcos.

new calculation at this time makes a significant improvement in the orbit quality, as illustrated by the decrease in formal errors for each of the elements. The distance and mass of the $\sigma$ Ori AB system are discussed in a recent paper by Caballero (2008).

There is no previous determination of orbital elements for SEE 322. While the errors and residuals are large, the orbital solution looks promising, although it is still preliminary since only a limited portion of the orbit is covered by existing data. The total mass associated with this solution is very large $\left(260 M_{\odot}\right.$ for a distance of $1.2 \mathrm{kpc}$; Mason et al. 1998), which may indicate that revisions are required in the assumed elements and distance, or that the system contains more than two stars.

\subsection{Single-Star Detections}

In addition to discovering new companions and measuring some parameters of previously known stars, we have a number of stars determined to be single under the specific observing conditions, AO system performance, and FOV. Table 4 lists these stars. In this table, the respective columns give the HD number, Hipparcos number, epoch of observation, FWHM of the corrected stellar PSF, and the V/C code for the spectroscopic binary status (see Section 3.1). A larger FWHM is indicative of poorer seeing, a dimmer primary, non-optimal AO system tuning, or some combination of these factors.

\subsubsection{Notes to Unresolved Systems}

$H D$ 15137. This runaway star is a probable, single-lined spectroscopic binary (Boyajian et al. 2005).

$H D$ 25638. The pair ES $2603(\rho \sim 6.15, \Delta m \sim 6.0)$ was not detected. It was probably just out of the FOV.
HD 30614. Classified as a runaway star in Gies \& Bolton (1986).

HD 36879. This star is a runaway object according to its proper motion (Mdzinarishvili \& Chargeishvili 2005).

$H D$ 37042. The known components to this star are all far too wide for detection here. The star $\theta^{2}$ Ori B is radial velocity variable according to Morrell \& Levato (1991).

$H D$ 37043. Iota Ori is a close speckle pair of great interest as, despite its very close separation, it was deemed optical in Gualandris et al. (2004). The speckle pair was last measured very close to the FWHM value, so it is possible that the component was just barely too close to be detected.

$H D$ 37366. This star is a double-lined spectroscopic binary (Boyajian et al. 2007b).

HD 39680. Classified as a runaway star in Gies \& Bolton (1986). The known pair, S 502, is much too wide (46". 1) for detection here.

HD 41161. The known pair, ES 1234AB, was too wide (10'3) for detection here. Whether this pair is bound or not is not yet certain.

HD 45314. CHR 251 was last measured closer (54 mas) than the FWHM of the PSF of our observation. The A component is an Oe star and a probable velocity variable (Boyajian et al. 2007a).

HD 52266. This star is a probable single-lined spectroscopic binary (McSwain et al. 2007).

HD 54662. This star is a double-lined spectroscopic binary (Boyajian et al. 2007b).

$H D$ 60848. This Oe star shows emission line variations but no evidence of significant velocity variability (Boyajian et al. 2007a). 
Table 4 Single-Star FWHM measures

\begin{tabular}{|c|c|c|c|c|}
\hline HD & HIP & $\begin{array}{l}\text { Epoch } \\
\text { (BY) }\end{array}$ & $\begin{array}{c}\text { FWHM } \\
\left({ }^{\prime \prime}\right)\end{array}$ & $\begin{array}{c}\text { Spectroscopic } \\
\text { status }^{\mathrm{a}}\end{array}$ \\
\hline 14947 & 11394 & 2001.7455 & 0.12 & $\mathrm{C}$ \\
\hline 15137 & 11473 & 2004.7923 & 0.18 & V \\
\hline 25638 & 19272 & 2004.8665 & 0.12 & $\mathrm{C}$ \\
\hline 30614 & 22783 & 2004.8665 & 0.12 & $\mathrm{C}$ \\
\hline 36879 & 26272 & 2003.8944 & 0.11 & $\mathrm{C}$ \\
\hline 37042 & & 2004.8667 & 0.12 & $\mathrm{~V}$ \\
\hline 37043 & 26241 & 2001.7455 & 0.08 & $\mathrm{~V}$ \\
\hline 37366 & 26611 & 2005.0200 & 0.34 & V \\
\hline 39680 & 27941 & 2002.2404 & 0.22 & $\mathrm{C}$ \\
\hline 41161 & 28881 & 2001.8659 & 0.16 & $\mathrm{C}$ \\
\hline 42088 & 29216 & 2001.8660 & 0.21 & $\mathrm{C}$ \\
\hline 45314 & 30722 & 2001.0989 & 0.11 & $\mathrm{~V}$ \\
\hline 46149 & 31128 & 2001.8660 & 0.36 & V \\
\hline 46966 & 31567 & 2002.2377 & 0.25 & $\mathrm{C}$ \\
\hline 48099 & 32067 & 2002.2405 & 0.13 & $\mathrm{~V}$ \\
\hline 52266 & 33723 & 2001.9754 & 0.12 & $\mathrm{~V}$ \\
\hline 53975 & 34297 & 2005.0196 & 0.12 & V \\
\hline \multirow[t]{2}{*}{54662} & 34536 & 2002.0218 & 0.16 & V \\
\hline & & 2002.2404 & 0.12 & \\
\hline 55879 & 34999 & 2005.0170 & 0.19 & $\mathrm{C}$ \\
\hline 57061 & 35415 & 2004.0807 & 0.21 & V \\
\hline 57682 & 35707 & 2001.9290 & 0.45 & $\mathrm{C}$ \\
\hline 60848 & 37074 & 2001.0990 & 0.16 & $\mathrm{C}$ \\
\hline 66811 & 39429 & 2004.0861 & 0.48 & $\mathrm{C}$ \\
\hline 69648 & & 2004.0451 & 0.26 & $\ldots$ \\
\hline 75211 & 43125 & 2004.1432 & 0.23 & $\ldots$ \\
\hline 75222 & 43158 & 2005.0197 & 0.49 & $\mathrm{C}$ \\
\hline 93521 & 52849 & 2001.0992 & 0.19 & $\mathrm{C}$ \\
\hline 148546 & 80829 & 2005.3482 & 0.28 & $\mathrm{C}$ \\
\hline 149404 & 81305 & 2001.3893 & 0.12 & V \\
\hline 149757 & 81377 & 2002.2243 & 0.22 & $\mathrm{C}$ \\
\hline 151003 & 82121 & 2004.2590 & 0.23 & $\mathrm{~V}$ \\
\hline 151515 & 82366 & 2004.3384 & 1.93 & $\mathrm{C}$ \\
\hline 152003 & & 2001.3893 & 0.12 & $\mathrm{C}$ \\
\hline 152219 & & 2004.5265 & 0.22 & $\mathrm{~V}$ \\
\hline 152314 & & 2001.4029 & 0.17 & $\mathrm{C}$ \\
\hline 153919 & 83499 & 2003.5247 & 0.16 & V \\
\hline 155806 & 84401 & 2002.6730 & 0.12 & $\mathrm{C}$ \\
\hline 157857 & 85331 & 2002.2383 & 0.22 & $\mathrm{C}$ \\
\hline 162978 & 87706 & 2001.7446 & 0.12 & $\mathrm{C}$ \\
\hline 163758 & & 2001.5179 & 0.17 & $\mathrm{C}$ \\
\hline \multirow[t]{2}{*}{163800} & 88040 & 2001.4960 & 0.13 & $\ldots$ \\
\hline & & 2001.5176 & 0.16 & \\
\hline 163892 & 88085 & 2005.3404 & 0.21 & V \\
\hline 164438 & 88297 & 2002.3204 & 0.16 & $\ldots$ \\
\hline 164794 & 88469 & 2002.6729 & 0.10 & V \\
\hline \multirow[t]{2}{*}{164816} & & 2001.6709 & 0.14 & V \\
\hline & & 2002.4817 & 0.11 & \\
\hline 165052 & 88581 & 2001.6711 & 0.14 & V \\
\hline 165319 & 88652 & 2001.6711 & 0.16 & $\mathrm{C}$ \\
\hline \multirow[t]{2}{*}{167771} & 89630 & 2002.4843 & 0.20 & $\mathrm{~V}$ \\
\hline & & 2002.4950 & 0.19 & \\
\hline 175876 & 93118 & 2001.7447 & 0.14 & $\mathrm{C}$ \\
\hline 186980 & 97280 & 2001.7366 & 0.19 & $\mathrm{C}$ \\
\hline \multirow[t]{2}{*}{188001} & 97796 & 2001.7338 & 0.12 & V \\
\hline & & 2001.7447 & 0.17 & \\
\hline 188209 & 97757 & 2004.7646 & 0.08 & $\mathrm{C}$ \\
\hline 190864 & 98976 & 2001.7365 & 0.14 & V \\
\hline 190918 & 99002 & 2001.7421 & 0.18 & V \\
\hline 191612 & 99308 & 2001.7365 & 0.13 & $\mathrm{C}$ \\
\hline 191978 & 99439 & 2002.6786 & 0.33 & $\mathrm{C}$ \\
\hline 192281 & 99580 & 2001.7365 & 0.12 & $\mathrm{C}$ \\
\hline 192639 & 99768 & 2001.7366 & 0.17 & $\mathrm{C}$ \\
\hline 193443 & 100146 & 2001.7366 & 0.19 & V \\
\hline
\end{tabular}

Table 4

(Continued)

\begin{tabular}{ccccc}
\hline \hline HD & HIP & $\begin{array}{c}\text { Epoch } \\
(\mathrm{BY})\end{array}$ & $\begin{array}{c}\text { FWHM } \\
\left({ }^{\prime \prime}\right)\end{array}$ & $\begin{array}{c}\text { Spectroscopic } \\
\text { status }^{\mathrm{a}}\end{array}$ \\
\hline 195592 & 101186 & 2005.6549 & 0.19 & $\mathrm{~V}$ \\
198846 & 102999 & 2005.3843 & 0.12 & $\mathrm{~V}$ \\
202124 & 104695 & 2005.6549 & 0.19 & $\mathrm{C}$ \\
203064 & 105186 & 2001.7342 & 0.12 & $\mathrm{C}$ \\
& & 2001.7448 & 0.12 & \\
207538 & 107598 & 2005.7180 & 0.22 & $\mathrm{C}$ \\
210809 & 109562 & 2001.7369 & 0.21 & $\mathrm{C}$ \\
210839 & 109556 & 2001.7342 & 0.16 & $\mathrm{C}$ \\
214680 & 111841 & 2001.7369 & 0.19 & $\mathrm{C}$ \\
216898 & 113218 & 2001.7451 & 0.10 & $\ldots$ \\
218915 & 114482 & 2004.7597 & 0.18 & $\mathrm{C}$ \\
\hline
\end{tabular}

Note. ${ }^{a}$ Codes are $\mathrm{V}$, one or more additional spectroscopic companions indicated or $\mathrm{C}$, radial velocity constant.

HD 69648. Not included in the Mason et al. (1998) survey. $H D$ 163892. These data were taken using an 800-900 nm filter.

$H D$ 164794. This star is a probable long-period spectroscopic binary (Rauw et al. 2002).

HD 167771. The known companion, RST 3170, last measured in 1940 at 8".3 (Rossiter 1955), would have been outside the FOV.

HD 175876. The known, probably optical, pair, HO 271, would have been too wide to detect.

HD 191978. Not included in the Mason et al. (1998) survey. The star shows no evidence of velocity variability (Abt et al. 1972).

$H D$ 193443. The close pair, A 1425AB $\left(\rho \sim 00^{\prime \prime} 1\right)$ may have been too close to resolve, while the wider AC pair $(\rho \sim 9.1)$ may have been outside the FOV.

$H D$ 195592. This is a probable single-lined spectroscopic binary (McSwain et al. 2007).

$H D$ 216898. Not included in the Mason et al. (1998) survey.

\subsection{MASS Data Mining Confirmations}

Searches were made for Two Micron All Sky Survey (2MASS) (Cutri et al. 2003) companions to all stars on our observing list, using Vizier and Aladin, as well as the "data mining" technique of Wycoff et al. (2006). Results are given in Table 5 and discussed below.

It should first be noted that the separation $/ \Delta \mathrm{m}$ regimes covered by these two techniques have rather limited overlap. The "plate scale" of the AO detector nominally limits us to companions within $\sim 5^{\prime \prime}$ of the primary, although this outer limit may extend to as wide as 7".5, depending on orientation of the pair relative to the detector, as well as slight off-center placement of the primary.

In order to get an idea of the separation $/ \Delta \mathrm{m}$ limits of 2MASS, the point-source catalog was searched for sources in the magnitude range $5.5 \leqslant J \leqslant 8.0$, corresponding to the approximate 2MASS $J$-magnitude range for the AO targets in this project. This yielded 99,656 sources. All sources within $10^{\prime \prime}$ of these "primaries" were then extracted from the catalog, yielding 9657 companions. A plot of separation versus $\Delta J$ is shown in Figure 6. As seen in this figure, very few close pairs are detected (only 17 pairs with separations in the range $1^{\prime \prime}<\rho<2^{\prime \prime}$ and an additional 70 in the range $2^{\prime \prime}<\rho<3^{\prime \prime}$ ). For the main body of companions, maximum $\Delta J$ increases to 


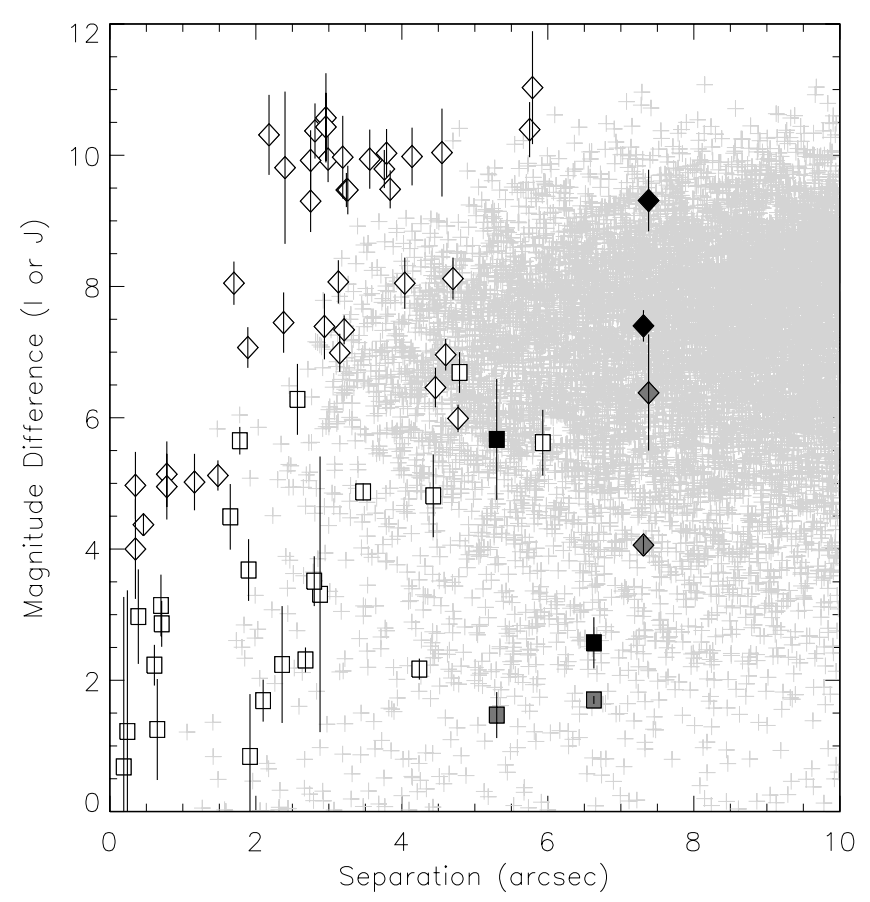

Figure 6. Magnitude difference versus separation from 2MASS and AO. AO observations are shown as squares (for previously-known pairs) or diamonds (for new pairs), with error bars plotted for the photometry only. The faint cloud of plus signs indicate all 2MASS pairs under $10^{\prime \prime}$ in separation, as described in the text. Two known and two new AO pairs were also found in the 2MASS data; these are shown as filled symbols, with 2MASS $J$-band magnitude differences in gray and $\mathrm{AO}$ magnitude differences in black. $V$-band magnitude differences for the two known pairs with AO and 2MASS data were very similar to the AO $\triangle m$ values, so are not plotted.

perhaps 6-6.5 mag at $3^{\prime \prime}, 8-8.8 \mathrm{mag}$ at $4^{\prime \prime}$, and 9.5-10 mag at $7 " .5$.

AO Single Stars. A Vizier search of the regions around each of the AO single stars found only six 2MASS companions within $7^{\prime \prime} .5$ of their respective primaries. HD 25638 (6".2), HD 52266 (7". 1), HD 151515 (6!.2), HD 152219 (7".2), HD 163892 (6."4), and HD 210809 (5."6). A further check of their orientations showed that all of these companions fell either outside the observing window or so close to the edge of the field as to not be measurable. The companion to HD 25638 is known; see Section 3.2.1.

Known Pairs. A search of the regions around these primaries found six 2MASS companions within 7".5. Two of these (HD 48279 and 152408) correspond to known companions also measured by $\mathrm{AO}$ and are listed in Table 5, which lists relative astrometry and differential photometry in the 2MASS $J, H$, and $K_{S}$ bands. The remaining four, including one known companion and three objects much wider than the known secondaries, all fall outside the $\mathrm{AO}$ observing window. Note that the companion to HD 152408 is very red $\left(\Delta m_{I}>\Delta m_{K_{S}}\right)$, perhaps indicating that the companion still possesses a luminous disk (like the case of the IR-bright companion of $\sigma$ Ori AB).

New Pairs. The search around these objects found five 2MASS companions within $7^{\prime \prime} .5$. Two of these (HD 156212 and 201345) correspond to the newly discovered AO companions and are listed in Table 5. The other three-two of which are known companions-are all wide.

\section{DISCUSSION}

Our AO survey of the O-stars has revealed a large number of new and generally faint companions. We show in Figure 7 the distribution of numbers of companions as a function of magnitude difference $\Delta I$ (in 1 mag bins), and the distribution peaks near $\Delta I=10 \mathrm{mag}$. It is certainly possible that some of these faint companions are low mass, gravitationally bound stars. For example, Hillenbrand (1997) made a deep I-band survey of the Orion cluster centered on the Orion Trapezium, and she found examples of still embedded pre-main-sequence stars with magnitude differences as large as $\Delta I=15 \mathrm{mag}$ compared to the bright $\mathrm{O}$-stars in the Trapezium. On the other hand, her survey also demonstrates that there will be many cluster stars that appear projected on the sky at positions near O-stars but are not orbitally bound to them.

We used the $I$-band star counts from Hillenbrand (1997) to model the possible confusion limit caused by nearby cluster stars that we might expect to find in our observations. The cumulative number distribution of stars brighter than $I$ in her sample (1551 stars with measured I magnitudes) is well matched by a power law,

$$
N(<I)=(0.063) 10^{\gamma I},
$$

where the exponent is $\gamma=0.27$ (valid down to $I=16 \mathrm{mag}$ ). The total surface density of stars in the central part of cluster where the O-stars reside is $10^{5.4}$ stars per square degree (see Figure 4a in Hillenbrand 1997) or 1.9 stars per $10^{\prime \prime} \times 10^{\prime \prime}$ FOV (= AEOS FOV). Thus, the cumulative distribution of cluster background stars per AEOS FOV is approximately given by

$$
N(<I)=\left(7.8 \times 10^{-5}\right) 10^{\gamma I} .
$$

If we suppose that all O-stars have a color index of $V-I=$ -0.39 (Wegner 1994), the extinction to Orion is approximately $A_{I}=0.25$ (Hillenbrand 1997; Fitzpatrick 1999), and the distance to Orion is $470 \mathrm{pc}$ (Hillenbrand 1997), then the numbers per AEOS FOV can be recast in terms of an O-star absolute magnitude $M_{V}$ and magnitude difference $\triangle I$ as

$$
N(<I)=(0.023) 10^{\gamma\left(\triangle I+M_{V}\right)} .
$$

If we further assume that the same relationship holds for other $\mathrm{O}$-stars in more distant clusters, then the number of cluster stars appearing in the FOV will scale as distance $d$ squared. Let $\eta$ represent the predicted number of cluster background stars per AEOS FOV. The relation between the limiting magnitude difference $\Delta I$ associated with $\eta$ is then

$$
\Delta I=3.6-\frac{2}{\gamma} \log d-M_{V}+\frac{1}{\gamma} \log \eta .
$$

Thus, we expect that background confusion with cluster stars will increase (smaller $\Delta I$ ) with increasing distance and with lower-luminosity O-stars.

We applied this relation to estimate a limiting $\Delta I$ for each cluster and the association star in our sample in order to separate the probable physical companions from the faint optical companions caused by background confusion. We used distances from Mason et al. (1998) and the spectral type-absolute magnitude calibration from Martins et al. (2005). The Orion cluster richness, extinction, and age may not be representative of the full sample of cluster O-stars in our survey, and so the normalization of the $\Delta I$ relation is somewhat uncertain. We note that large numbers of companions begin to appear near $\Delta I=$ $8 \mathrm{mag}$ (see Figure 7), and for typical O-star distances $(d=1$ to $2 \mathrm{kpc})$ and magnitudes $\left(M_{V}=-4\right.$ to -6$)$ this corresponds 


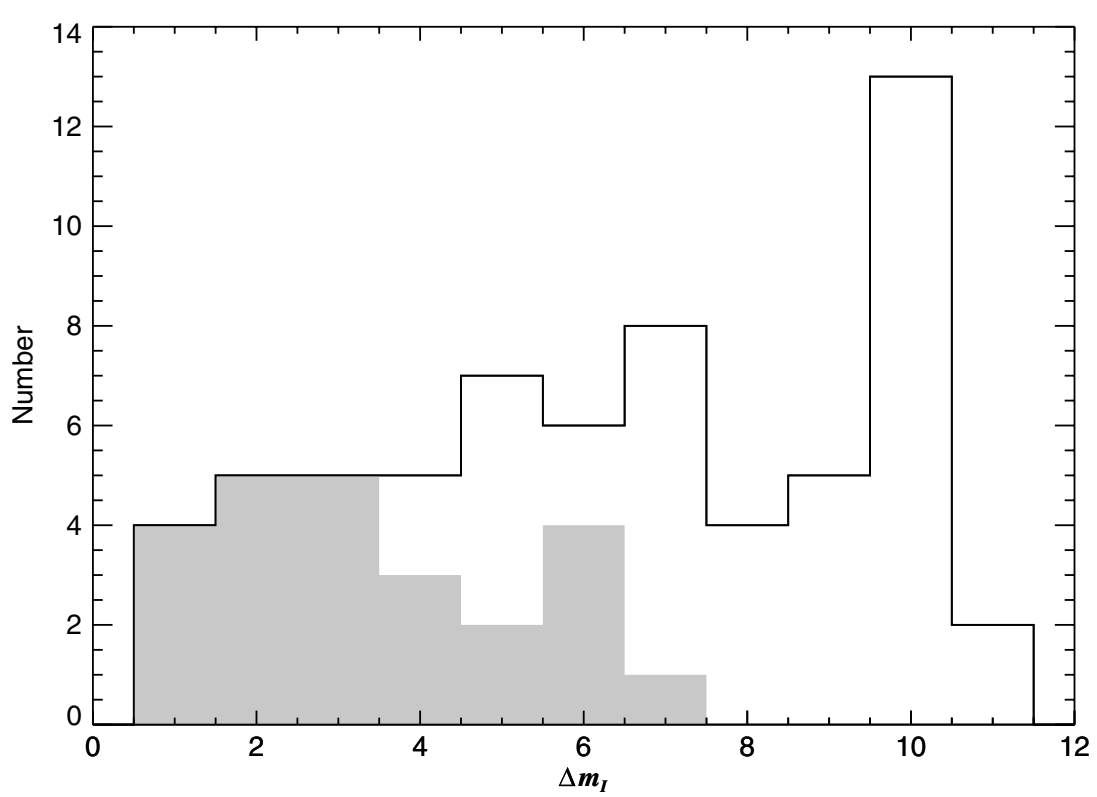

Figure 7. Number of companions vs. differential magnitude at $I$-band. Each bin represents a magnitude difference of 1 mag. The shaded region represents previously known companions while the solid line histogram shows the total number of companions (known plus new).

Table 5

Data Mining Results from 2MASS

\begin{tabular}{lrrccccccc}
\hline \hline Discoverer designation & \multicolumn{1}{c}{ HD } & \multicolumn{1}{c}{ HIP } & WDS & Epoch (BY) & P.A. $\left({ }^{\circ}\right)$ & Separation $\left({ }^{\prime \prime}\right)$ & $\Delta \mathrm{m}_{J}(\mathrm{mag})$ & $\Delta \mathrm{m}_{H}(\mathrm{mag})$ & $\Delta \mathrm{m}_{K s}(\mathrm{mag})$ \\
\hline STF 956 AB & 48279 & 32137 & $06427+0143$ & 1999.87 & 194.8 & 6.55 & $1.70 \pm 0.06$ & $1.63 \pm 0.03$ & $1.56 \pm 0.04$ \\
I 576 & 152408 & 82775 & $16550-4109$ & 1999.36 & 264.2 & 4.72 & $1.47 \pm 0.35$ & $1.71 \pm 0.58$ & $1.25 \pm 0.44$ \\
TRN 24 AC & 156212 & 84588 & $17175-2746$ & 1998.53 & 147.6 & 7.32 & 4.06 & $5.38 \pm 0.14$ & 4.24 \\
TRN 31 & 201345 & 104316 & $21079+3324$ & 1999.75 & 220.8 & 7.44 & $6.38 \pm 0.89$ & $5.88 \pm 0.45$ & $5.69 \pm 0.24$ \\
\hline
\end{tabular}

Table 6

Companion Frequency and Environment

\begin{tabular}{lcccc}
\hline \hline Category & $\begin{array}{c}\text { Number } \\
\text { of stars }\end{array}$ & $\begin{array}{c}\text { Number } \\
\text { w/companion }\end{array}$ & $\begin{array}{c}\text { Number } \\
\text { w/physical companion }\end{array}$ & $\begin{array}{c}\text { Percentage } \\
\text { of physical companion }\end{array}$ \\
\hline Cluster/association & 83 & 41 & 31 & $37 \pm 7$ \\
Field & 9 & 2 & 1 & $11 \pm 11$ \\
Runaway & 24 & 8 & 1 & $4 \pm 4$ \\
\hline
\end{tabular}

to a background density in the Orion model of $\eta=1$ star per AEOS FOV. Thus, we adopted this value of $\eta$ in Equation (4) to identify the probable physical companions among the cluster and association stars.

The background limits for the field and runaway stars depend instead upon on the stellar number density of Galactic background stars as a function of magnitude and the direction of a given target. Here we adopted the cumulative star counts as a function of apparent $I$ magnitude and Galactic coordinates from the Besançon model of the Galaxy (Robin et al. 2003). We created a cumulative star counts versus $\Delta I$ relation for each of these stars and found a limiting magnitude for a specified value of 0.01 stars per AEOS FOV. We set this conservative limit based on numerical experiments for the set of runaway stars with observed faint companions. None of these companions can be physical companions since any such wide binary would be disrupted at the time the runaway star was ejected (by close gravitational encounters or a supernova in a binary).

The results of this exercise to discern the probable physical companions are summarized in Table 6 . The targets were placed in categories of cluster and association stars, field stars, and runaway stars according to the criteria adopted by Mason et al.
(1998) (with some revisions from the subsequent work on field O-stars by de Wit et al. 2004, 2005). We see that after rejection of the possible background stars there is still a high frequency (37\%) of detected companions among the cluster and association stars in our survey. In sharp contrast, there are very few such companions among the field and runaway stars. The only field star with a probable companion is HD 48279 , and de Wit et al. (2004) show that this star is $28^{\prime \prime}$ from a cluster identified in 2MASS images, which suggests that this O-star may be a member of a young, emerging cluster. The only runaway star with a candidate companion is HD $34078=$ AE Aur, a star ejected from the Orion association (Gualandris et al. 2004). There is another optical companion, SEI 136, at a separation of 8."4 (Mason et al. 1998), and we suspect that both the companion we found and SEI 136 form a visual system that appears by a chance alignment with AE Aur at this time in its trajectory across the sky.

Our results confirm the trends found by Mason et al. (1998) that binary O-stars are common among O-stars found near their birthplaces and are rare among $\mathrm{O}$-stars ejected from clusters. These trends are consistent with current models for the ejection of runaways and the formation of massive stars (Zinnecker 
\& Yorke 2007). High-velocity O-stars were probably ejected by close encounters between binaries in dense clusters and by supernova explosions in close binaries (Hoogerwerf et al. 2001), and their ejection velocities generally exceed the escape velocity binding any wide, orbiting companion. Many massive stars are probably born in high-density stellar environments where binaries may be formed through three-body interactions (Bate et al. 2002) and through the interactions with large proto-stellar accretion disks (Moeckel \& Bally 2007). Even in lower-density environments, binaries may form through disk fragmentation and subsequent gas accretion (Kratter et al. 2007). Our results add to growing body of evidence that the formation of binaries is closely linked to the formation of massive stars and the deposition of the angular momentum reservoir of the natal cloud.

The United States Air Force provided the telescope time, onsite support, and $80 \%$ of the research funds for this Air Force Office of Scientific Research and National Science Foundation (NSF) jointly sponsored research under NSF grant number AST0088498. Additional support was provided by NSF grants AST0506573 and AST-0606861. L.C.R. was funded by AFRL/DE (Contract Number F29601-00-D-0204). We thank the numerous staff members of the Maui Space Surveillance System who helped make these data possible. Thanks as well to Gary Wycoff of USNO, who "mined" the 2MASS Catalog for additional photometry and astrometry of these systems. This research has made use of the Washington Double Star Catalog, maintained at the U.S. Naval Observatory, and of Aladin, SIMBAD, and Vizier, operated at CDS, Strasbourg, France. This publication also makes use of data products from the Two Micron All Sky Survey, which is a joint project of the University of Massachusetts and the Infrared Processing and Analysis Center/California Institute of Technology, funded by the National Aeronautics and Space Administration and the National Science Foundation.

\section{REFERENCES}

Abt, H. A., Levy, S. G., \& Gandet, T. L. 1972, AJ, 77, 138

Africano, J. L., Cobb, C. L., Dunham, D. W., Evans, D. S., Fekel, F. C., \& Vogt, S. S. 1975, AJ, 80, 689

Bate, M. R., Bonnell, I. A., \& Bromm, V. 2002, MNRAS, 336, 705

Boyajian, T. S., et al. 2005, ApJ, 621, 978

Boyajian, T. S., et al. 2007a, PASP, 119, 742

Boyajian, T. S., et al. 2007b, ApJ, 664, 1121

Caballero, J. A. 2007, A\&A, 466, 917

Caballero, J. A. 2008, MNRAS, 383, 750

Cox, A. N. 2001, Allen's Astrophysical Quantities (4th ed.; New York: Springer) Cutri, R. M., et al. 2003, 2MASS All Sky Catalog of Point Sources (Pasadena, CA: NASA/IPAC)

de Wit, W. J., Testi, L., Palla, F., Vanzi, L., \& Zinnecker, H. 2004, A\&A, 425, 937

de Wit, W. J., Testi, L., Palla, F., \& Zinnecker, H. 2005, A\&A, 437, 247

Fabricius, C., \& Makarov, V. V. 2000, A\&A, 356, 141

Fitzpatrick, E. L. 1999, PASP, 111, 63

Gies, D. R., \& Bolton, C. T. 1986, ApJS, 61, 419

Gualandris, A., Portegies Zwart, S., \& Eggleton, P. P. 2004, MNRAS, 350, 615

Hartkopf, W. I., Mason, B. D., \& McAlister, H. A. 1996, AJ, 111, 370
Hartkopf, W. I., Mason, B. D., \& Worley, C. E. 2001, AJ, 122, 3472

Hillenbrand, L. A. 1997, AJ, 113, 1733

Hillwig, T. C., Gies, D. R., Bagnuolo, Jr., W. G., Huang, W., McSwain, M. V., \& Wingert, D. W. 2006, ApJ, 639, 1069

Hinkley, S., et al. 2007, ApJ, 654, 633

Hoogerwerf, R., de Bruijne, J. H. J., \& de Zeeuw, P. T. 2001, A\&A, 365, 49

Hopmann, J. 1967, Mitteilungen der Universitaets-Sternwarte Wien, 13, 49

Hummel, C. A., White, N. M., Elias, II, N. M., Hajian, A. R., \& Nordgren, T. E. 2000, ApJ, 540, L91

Kratter, K. M., Matzner, C. D., \& Krumholz, M. R. 2007, arXiv:0709.4252

Kraus, S., et al. 2007, A\&A, 466, 649

Maíz-Apellániz, J., Walborn, N. R., Galué, H. A., \& Wei, L. 2004, ApJS, 151,103

Makidon, R. B., Sivaramakrishnan, A., Perrin, M. D., Roberts, L. C., Jr., Oppenheimer, B. R., Soummer, R., \& Graham, J. R. 2005, PASP, 117, 831

Martins, F., Schaerer, D., \& Hillier, D. J. 2005, A\&A, 436, 1049

Mason, B. D., Hartkopf, W. I., Gies, D. R., Bagnuolo, Jr., W. G., ten Brummelaar, T. A., \& McAlister, H. A. 1998, AJ, 115, 821

Mason, B. D., Hartkopf, W. I., Holdenried, E. R., \& Rafferty, T. J. 2001a, AJ, 121,3224

Mason, B. D., Wycoff, G. L., Hartkopf, W. I., Douglass, G. G., \& Worley, C. E. 2001b, AJ, 122, 3466

Massey, P., Armandroff, T. E., Pyke, R., Patel, K., \& Wilson, C. D. 1995, AJ, 110,2715

McSwain, M. V. 2003, ApJ, 595, 1124

McSwain, M. V., Boyajian, T. S., Grundstrom, E. D., \& Gies, D. R. 2007, ApJ, 655,473

Mdzinarishvili, T. G., \& Chargeishvili, K. B. 2005, A\&A, 431, L1

Moeckel, N., \& Bally, J. 2007, ApJ, 661, L183

Morrell, N., \& Levato, H. 1991, ApJS, 75, 965

Otero, S. A. 2007, Open Eur. J. Variable Stars, 72, 1

Perryman, M. A. C., \& ESA 1997, The Hipparcos and Tycho Catalogues. Astrometric and Photometric Star Catalogues Derived from the ESA Hipparcos Space Astrometry Mission, ESA SP Series Vol. 1200 (Noordwijk: ESA Pub. Div.)

Pozzo, M., Jeffries, R. D., Naylor, T., Totten, E. J., Harmer, S., \& Kenyon, M. 2000, MNRAS, 313, L23

Rauw, G., et al. 2002, A\&A, 394, 993

Roberts, Jr., L. C. 2001, in Proc. 2001 AMOS Technical Conference, ed. P. Kervin, L. Bragg, \& S. Ryan (Maui, HI: Maui Econ. Devel. Board), 326

Roberts, Jr., L. C., \& Neyman, C. R. 2002, PASP, 114, 1260

Roberts, Jr., L. C., Turner, N. H., \& ten Brummelaar, T. A. 2007, AJ, 133, 545

Robin, A. C., Reylé, C., Derrière, S., \& Picaud, S. 2003, A\&A, 409, 523

Rossiter, R. A. 1955, Publ. Michigan Obs., 11, 1

See, T. J. J. 1898, AJ, 18, 181

Seymour, D. M., Mason, B. D., Hartkopf, W. I., \& Wycoff, G. L. 2002, AJ, 123,1023

Silbernagel, E. 1931, Astron. Nachr., 241, 33

Struve, F. G. W. 1837, Astron. Nachr., 14, 249

ten Brummelaar, T. A., Hartkopf, W. I., McAlister, H. A., Mason, B. D., Roberts, Jr., L. C., \& Turner, N. H. 1998, in Proc. SPIE, Adaptive Optical System Technologies, ed. D. Bonaccini, \& R. K. Tyson (Bellingham, WA: SPIE), 3353, 391

ten Brummelaar, T. A., Mason, B. D., Bagnuolo, Jr., W. G., Hartkopf, W. I., McAlister, H. A., \& Turner, N. H. 1996, AJ, 112, 1180

ten Brummelaar, T. A., Mason, B. D., McAlister, H. A., Roberts, Jr., L. C., Turner, N. H., Hartkopf, W. I., \& Bagnuolo, Jr., W. G. 2000, AJ, 119,2403

van den Bos, W. H. 1928, Ann. Leiden Obs., 14, pt. 4

van Loon, J. T., \& Oliveira, J. M. 2003, A\&A, 405, L33

Wallenquist, A. 1934, Ann. Bosscha Obs. Lembang, 6, pt. 2

Wegner, W. 1994, MNRAS, 270, 229

Worley, C. E. 1990, in Errors, Bias and Uncertainties in Astronomy, ed. C. Jaschek, \& F. Murtagh (Cambridge: Cambridge Univ. Press), 419

Wycoff, G. L., Mason, B. D., \& Urban, S. E. 2006, AJ, 132, 50

Zinnecker, H., \& Yorke, H. W. 2007, ARA\&A, 45, 481 